\title{
Unfolding - A library for interactive maps
}

\author{
Till Nagel ${ }^{1,2}$, Joris Klerkx ${ }^{2}$, Andrew Vande Moere ${ }^{3}$, and Erik Duval ${ }^{2}$ \\ 1 Interaction Design Lab, FH Potsdam \\ mail@tillnagel.com \\ 2 Department of Computer Science, KU Leuven \\ \{joris.klerkx, erik.duval\}@cs.kuleuven.be \\ 3 Department of Architecture, Urbanism and Planning, KU Leuven \\ andrew . vandemoere@asro.kuleuven. be
}

\begin{abstract}
Visualizing data with geo-spatial properties has become more important and prevalent due to the wide spread dissemination of devices, sensors, databases, and services with references to the physical world. Yet, with existing tools it is often difficult to create interactive geovisualizations tailored for a particular domain or a specific dataset. We present Unfolding, a library for interactive maps and data visualization. Unfolding provides an API for designers to quickly create and customize geo-visualizations. In this paper, we describe the design criteria, the development process, and the functionalities of Unfolding. We demonstrate its versatility in use through a collection of examples. Results from a user survey suggests programmers find the library easy to learn and to use.
\end{abstract}

Keywords: toolkits, maps, geovisualization, information visualization, interaction design, programming

\section{Introduction}

Until the extensive digitalization of geo-spatial data, cartographic products have been nearly exclusively created by cartographers, geographers, and scientists from other disciplines with a spatial context. Nowadays, interactive maps and geo-visualizations are prevalent on the internet, on navigation devices and smartphones, as well as on large-scale multitouch displays in exhibitions and public spaces. Similarly, interactive or animated maps are used increasingly to communicate facts or stories related to geo-spatial information in various application domains [1].

Areas are ranging from social networks to mobility patterns to data journalism to many more. For example, Dodge et al [2] argue that there is a "spatial turn" in social sciences, and that researchers are exploiting the geo-spatial components of large data to understand spatial relations and interactions. They describe interactive geographic visualization as an essential research tool. MoMA Design curator Antonelli sees visualization as one of the central design disciplines [3], and demonstrates current 


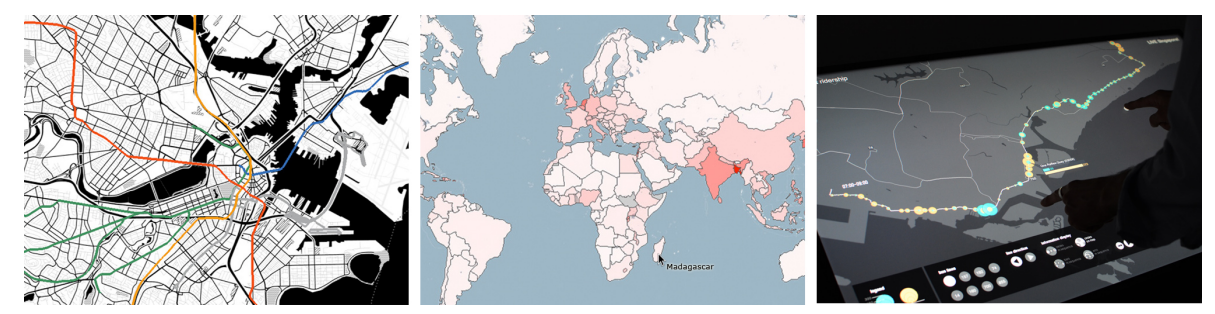

Fig. 1. Three applications created with Unfolding: An animated map showing subways in Boston (left), an interactive choropleth map showing population density (middle), and a visualization showing ridership in Singapore for a multitouch tabletop (right)

trends with eight examples of "highest quality of design", of which six use geo-spatial data visualized on maps. Moreover, there is an increase in the interest of the general public, among others due to the wide-spread use of location based apps for smartphones. Due to the recent 'creativity boom', in which "novel types of graphic [...] and interaction were being applied to new data and new scenarios" [4], different user groups should be encouraged to experiment in the geovisualization field.

But while interactive geovisualization is useful in a variety of domains, the tools that can be employed to generate the visualizations are either cumbersome to use, or lack the appropriate functionalities. There is an increasing need for interactive maps and geovisualizations, and many amateurs and non-GIS researchers are now creating and customizing geospatial data representations. In order to support a democratization of tools and technology we developed the Unfolding library.

With our library we strive to support three different purposes, i.e. (i) having a simple API that is easy to learn and use, (ii) support creating prototypes to quickly visualize data and to rapidly test novel interaction techniques, and (iii) building applications for a broader audience. We chose to implement Unfolding as a Processing library, and are going to introduce Processing and explain our reasoning for choosing it in Section 4.3. Since its inception, Unfolding has been used in course assignments, research projects and commercial products. The iterative development of Unfolding was guided by the needs of, and with constant feedback from the library users.

The remainder of this paper is structured as following: We give an overview on related work (Section 2), and describe our design goals (Section 3). In Section 4 we introduce the Unfolding library, its interaction and visualization features, and our design rationales. We demonstrate its 
usefulness with exemplary applications for each of the three purposes, and summarize the results of a user survey in Section 5.

\section{Related Work}

In the following we examine software and tools for the creation of interactive geovisualizations. We describe their different goals, and how they are only partly fitting for the purposes we aim to support. We discuss their advantages and drawbacks, and describe how they differ from Unfolding.

\subsection{GIS software}

Standard geographic information systems (GIS) foremost aim is to support analyzing geospatial data, but often do not allow extensive adaptation and simplification for a non-GIS-experts audience. Researchers in the field of geovisual analytics have argued that software "should be lightweight, easily deployable and usable, rather than huge and complex like current GIS." [5]. There is the need for less complex software which encourages interactivity [6], and supports interactions facilitating a knowledge construction process [7].

Thanks to easier tools for creating customized maps since the release of Google Maps and other web mapping services there are more and more interactive maps created by persons who have no expertise in GIS. This kind of map mashups needs little or no programming to visualize information spatially [8]. While such mashups often only include dots on a map or other basic display techniques, the mashup principle of re-using existing technology has been described as a means to rapidly create prototypes for geovisualization [9]. Simpler web-based GIS applications support selected visualization (e.g. IndieMapper [10]) or interaction techniques (e.g. GeoCommons [11]), and customization to a certain degree.

Generalized, GIS software facilitates in-depth analytics, but is complex, has a high learning curve, and is intended for experts. Mashup tools are easy-to-use, suitable for quick data exploration, and intended for nonexperts, but only allow employing a fixed set of techniques. An approach to fill this gap is the geoviz toolkit [12], which offers a graphical user interface (GUI) for users without programming expertise, yet it targets an audience of GIS experts.

Effective geo-visualizations employ established techniques, but tailor the visualization to the application domain and to the specific dataset. Thus, custom visualizations have to be created with toolkits or with software libraries. 


\subsection{Visualization and map libraries}

The Java GIS library GeoTools provides extensive functionality for geospatial data, and aims to support developing complex spatial data processing applications [13], but is targeted to professional software developers.

In recent years, multiple libraries have been published with the intent to allow designers and web developers to create interactive visualizations. As we are not aware of surveys on modern visualization libraries aimed at these new user groups, we chose an online collection [14], of which 12 out of 43 tools include map or other geo-spatial components. Data visualization libraries such as d3 [15] or Prefuse [16] aim to supporting general purpose visualizations and include a broad spectrum of techniques. With this, however, they tend to not focus on the geospatial area.

Dedicated map libraries such as Leaflet [17] or Polymaps [18] offer functionality to create interactive maps, and display geo-spatial data. These libraries have proven value in practice, which is also why design and functionality of Unfolding were guided by them. However, they are intended exclusively for a web environment, and thus only partially support more advanced interactive applications such as exhibits for large multitouch devices. Furthermore, they are not developed for the Processing environment, which prevents the usage in existing Processing projects, and reduces the applicability for less advanced users (see Section 4.3).

We are aware of three libraries providing basic map functionality for Processing. Their purpose is to provide rudimentary mapping features: all of them offer the display of a geo-referenced map, with conversion methods between geo-locations and Cartesian screen coordinates and vice versa. The geomap library by giCentre [19] provides functionality to load and display Shapefiles, a standard file format for GIS data. It allows interactive feature picking, and color coding, e.g. for choropleth maps (a thematic map with its areas shaded according to a data value). Google Mapper [20] allows downloading a Google map section and storing it as single image. Unlike Unfolding, none of these Processing libraries provide zooming and panning, dynamic map tile handling, an event system, multiple coordinates map views, additional geo data loading, or custom markers. Lastly, ModestMaps [21] is an extensive map and geovisualization JavaScript library for the web, for which a port to Processing was created in 2008. The main JavaScript library has many of the features missing in the other Processing map libraries, but the port for Processing is not actively developed, and only supports some of the basics. However, the tile-handling mechanism was mature and feature rich, which we therefore used as basis for Unfolding's own tile-handling functionality. 


\section{Design Goals}

This section introduces the design goals of Unfolding, and how the library enables developers ${ }^{4}$ (i) to easily create simple sketches ${ }^{5}$ with interactive maps, (ii) to quickly implement prototypes, and (iii) to create sophisticated visualizations, or even extend Unfolding's functionality.

For these purposes, Unfolding was developed with the main goals of learnability, simplicity, and extensibility. To support the first goal, the library comes with extensive documentation, mostly in the form of tutorials and example code. The documentation can be found both online at unfoldingmaps.org as well as in the downloadable distribution. The library uses a simple programming interface (API) to support the second goal. Library users can create interactive maps in very few lines of code (see Code sample 1). And thirdly, the library provides reusable components, and employs a software architecture allowing to extend its functionality in order to create advanced visualizations.

\subsection{Task areas}

We identified design goals and requirements, based on the experience from our own design projects, from our teaching, and collected as feedback from external users of the library, and grouped them into three main task areas. These groups partially converge, and are not necessarily strictly disjoint, but are useful nonetheless to refer back to and to describe how we aim to support the dominant tasks of the target audience. We describe the activities, user groups, and typical use cases.

i Learning Includes all activities in which developers learn how to display geo-spatial data. Users in this group mostly create simple sketches where they show markers on an interactive map. They use it for experiments and small projects.

ii Prototyping Includes all activities in which library users explore and understand geo-spatial data in an iterative data visualization design process. This also includes to quickly prototype sketches to try out new visualization or interaction ideas. Developers include both beginners and advanced users.

\footnotetext{
4 This paper differentiates between developers or library users for Unfolding library developers, and end users for persons using applications created with Unfolding.

${ }^{5}$ This paper uses the term sketch as introduced by Reas and Fry [22] where small programs act as software sketchbook allowing to quickly explore different ideas.
} 
iii Creating Includes all activities in which library users create larger projects. This can be for design studies by researchers to be able to evaluate novel techniques. This also can be for commercial or art projects where developers create complex geovisualizations.

All library users - that is persons creating visualizations or interactive applications with Unfolding - must have programming skills, ranging from beginner (learning) to intermediate and expert level (prototyping and creating). All of them have in common, that they not necessarily have expert geography or cartography knowledge.

Overall, Unfolding is developed to have a gentle learning curve, i.e. empowering to create standard visualizations in a few lines of code, and to create more complex visualizations when users are accustomed and more experienced with the library.

\subsection{Design process of Unfolding}

Since the first version of Unfolding in 2008, we continuously gathered feedback from library users. In the process of designing Unfolding its functionality was based on the lessons learnt from class room usage, and on the requirements of our own case studies in visualization. We follow the argument of Heer et al [16], and see iterative development, an established method for designing HCI, to be also a valuable design process for software libraries. In this vein, we discuss how the utilization of Unfolding in each task group helped the progress of Unfolding, and how the feedback from developers with different expertise levels helped us to balance learnability and functionality.

Learning. Since 2009 Unfolding has been used in six courses at Fachhochschule Potsdam, and two at IUAV University of Venice by the authors. Besides, it has been endorsed in various coursed at international universities (e.g. Carnegie Mellon, ITP, MIT), with departments ranging from interaction design to computer science to urban studies. The use in courses and workshops for teaching basics on geospatial data visualization allowed us to observe how beginners were using the library, and simplify the API and improve the documentation.

Prototyping. In early stages of designing an application, quick visual representations of geo-spatial data help to understand them. While these data loading and visualization methods can be implemented with other software or libraries, Unfolding provides them too in order to support library users all the way from learning up to creating. In addition, users have employed Unfolding to create geovisualizations with the purpose 
to prototype and evaluate new techniques. Unfolding aims to bridge the gap between traditional and novel visualizations by easing the creation of rapid design experiments.

Creating. Comments and suggestions from advanced users employing Unfolding in research, design, and commercial projects helped us refine existing and add frequently demanded features. Furthermore, successful design projects can act as flagship and inspire new groups of users.

\section{The Unfolding library}

This section introduces Unfolding, describes some of the basic interactions and visualizations methods, demonstrate its features and usage by examples, and explains our design rationales.

The code samples in 4.1 are to demonstrate the usage of the library in order to implement some of the fundamental functionality. This is to show how the basics are achievable in just a few lines of code, as stated in one of our design goals. For longer code examples we deem a paper not as the most appropriate form, and refer to the example section on our web page.

\subsection{Interaction \& Visualization}

Unfolding supports basic techniques for interactive maps such as zoom and pan, but also other common but slightly more advanced techniques such as Overview+Detail, i.e. showing a large scale map view while keeping the context by displaying the selected region on a large scale map.

Basic map. In just three lines of code library users can create an interactive map. The map is displayed in a default style with cartographic data from OpenStreetMap [23] and tiles from CloudMade [24].

UnfoldingMap map = new UnfoldingMap(this);

MapUtils.createDefaultEventDispatcher(this, map);

map.draw ();

Code sample 1. Creating an interactive map in Unfolding

To use another map style, developers can specify a different provider as second parameter when creating an UnfoldingMap. Our library provides eight pre-configured map tile providers for educational purposes. Developers can also create their own map provider to use customized map styles 
adapted to the requirements of their visualization. For instance, if the objective of the map is to support general spatial recognition while being discreet enough to not hinder the display of the data and interface layers, a minimal style with selected geographical features could be employed.

Basic interactions. By creating the default event dispatcher (as shown above), end users already can interact with the map. They can pan the map by dragging it with the mouse, or by using the arrow keys on the keyboard. Using the mouse wheel zooms in or out, which also works by pressing + or - keys. Double-clicking on the map centers it around that location, and zooms in one level. These basic interaction patterns were based on studies for map interactions ([25], [26]) and well-established design patterns for navigating and browsing [27].

Basic interactions with markers, i.e. visual representations of geographic features or data entries, are also provided out of the box. These include selecting and highlighting markers by clicking or tapping on them. More sophisticated interactions such as brushing and linking have to be implemented by the developers, but can employ Unfolding's event mechanism.

Multitouch interactions. Unfolding also provides interaction handling for multitouch devices. To turn on this feature developers have to register Unfolding's multitouch handler, which maps gesture input to map manipulation methods.

We focused on simple navigation patterns (e.g. pinch to zoom, drag to pan, tap to select) to support end users interact with the maps in ways more laymen have experience with, due to the wide-spread dissemination of smartphones and tablet computers with multitouch capabilities.

Visualizing data on a large-scale multi-touch surface allows the application of natural interaction techniques to engage a broad audience. Unfolding supports a high fluidity of the visualizations, with smooth transitions and low responsive times, in order to create enjoyable user experiences. See the project descriptions in Section 4.2 for examples of visualizations on multitouch tables.

Visualization features. Developers can use Unfolding's built-in marker mechanism to display geo-spatial data on the map. When end users interactively change the map area, or when the map is animated, latitude and longitude of the locations are converted to the correct screen positions, in the background. 
Location berlinLocation $=$ new Location(52.5, 13.4)

Marker berlinMarker = new SimplePointMarker(berlinLocation); map.addMarker (berlinMarker);

Code sample 2. Adding a location marker to display

Unfolding provides a default marker style, and has point, line, and polygon markers out of the box. Besides these markers, developers can also create multiple markers consisting of two or more markers of any kind, or use various connections representing some relationship between markers.

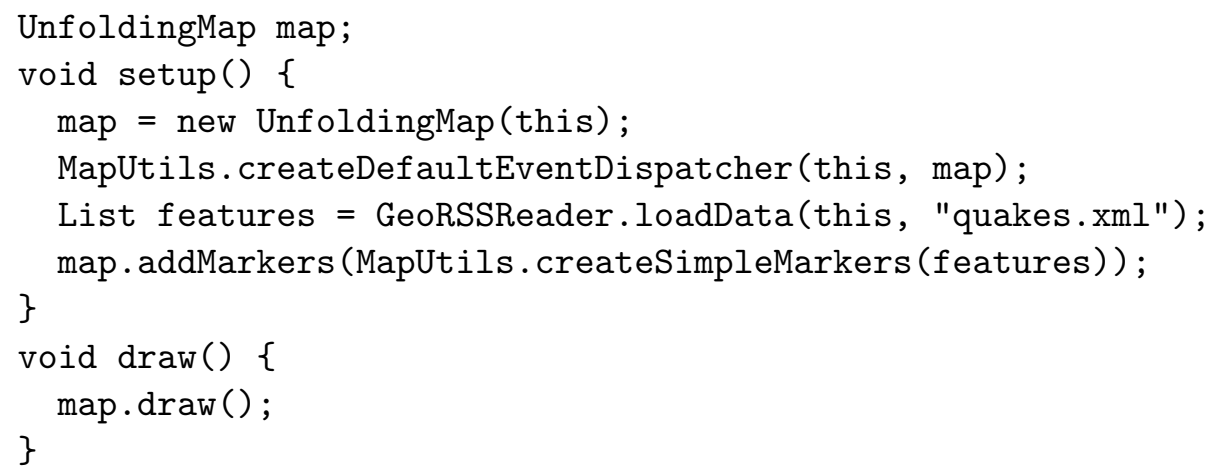

Code sample 3. A Processing sketch loading and displaying earthquakes on an interactive map. The earthquake data comes from the U.S. Geological Survey institution provided in the GeoRSS format

The library also allows reading standard formats for geospatial data, and automatically creating the respective graphical representations. The provided data readers support basic functionality, and do not fully implement the respective specifications. The GeoJSON parser supports most features, while the GeoRSS reader supports only Simple and W3C Geo, but not GML, and the GPX reader only enables reading track points. The aim was not to re-implement functionality developers can use and integrate from more sophisticated GIS libraries, but to enable getting quick results in a rapid prototyping approach. By building upon the Processing framework, developers can easily create own data readers. For example, Fig. 1(left) shows the display of subway lines in Boston, in which the geospatial routes as well as the train schedules comes from General Transit Feed Specification (GTFS) files provided by the transport authority.

The marker style can be customized, or completely implemented anew by the designers. The second option allows using data glyphs such as 
donut charts or any other data display technique (see Fig. 3). By mapping a value to the brightness value of a polygon marker, one can create simple choropleth maps. The example in Fig. 1(middle) shows an interactive version displaying population density of the world. End users can select single countries by hovering over (one of) the country's polygons, and additional data is display on demand.

\subsection{Example projects}

The following two Unfolding projects were selected to represent the spectrum of how the library can be used, and to exemplify various advanced features of Unfolding.
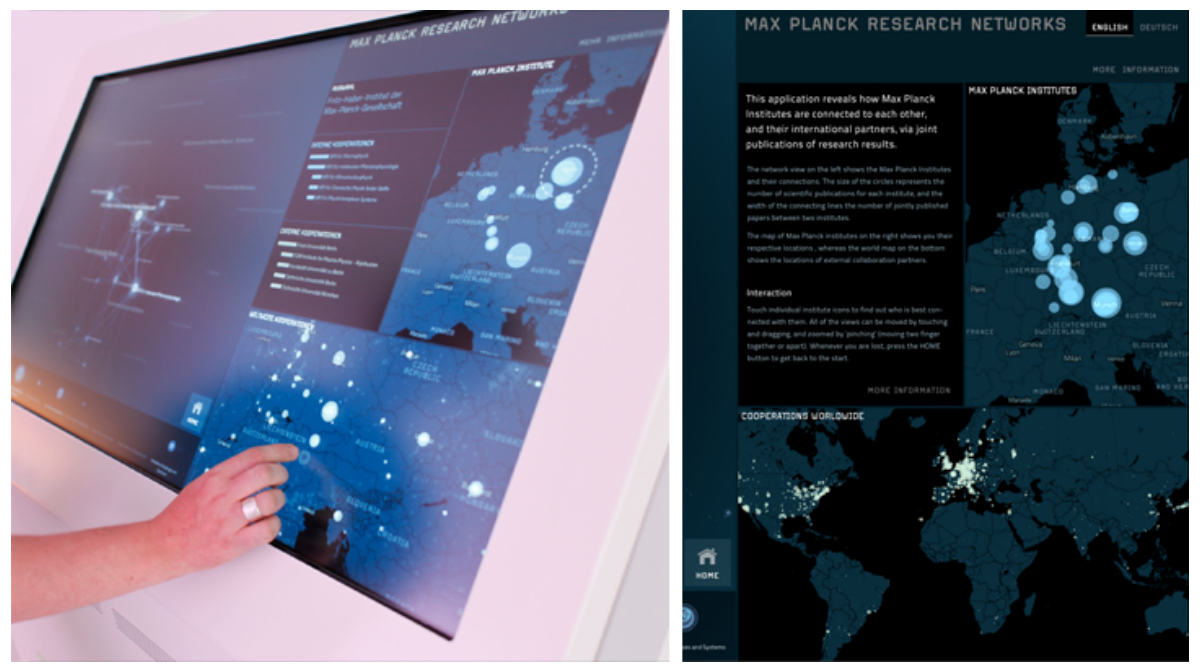

Fig. 2. Visualization of research networks on a multitouch table (left) with two Unfolding maps showing institutions (clipping right)

Max-Planck-Research Networks. A visualization of research networks on a multitouch table [28]. It uses three coordinated multiple views: one showing a network with institutions and their connections based on co-published papers, and two maps showing the locations of institutions in Germany and the world (see Fig. 2). Tapping on an institution in any view highlights it in all other views. The maps are implemented with Unfolding, and use custom styled map tiles. The application uses Unfolding's multitouch capabilities in order to allow end users to slide for panning 
and to pinch for zooming the maps. Brushing and linking interactions can be developed with Unfolding's event system to coordinate multiple maps.

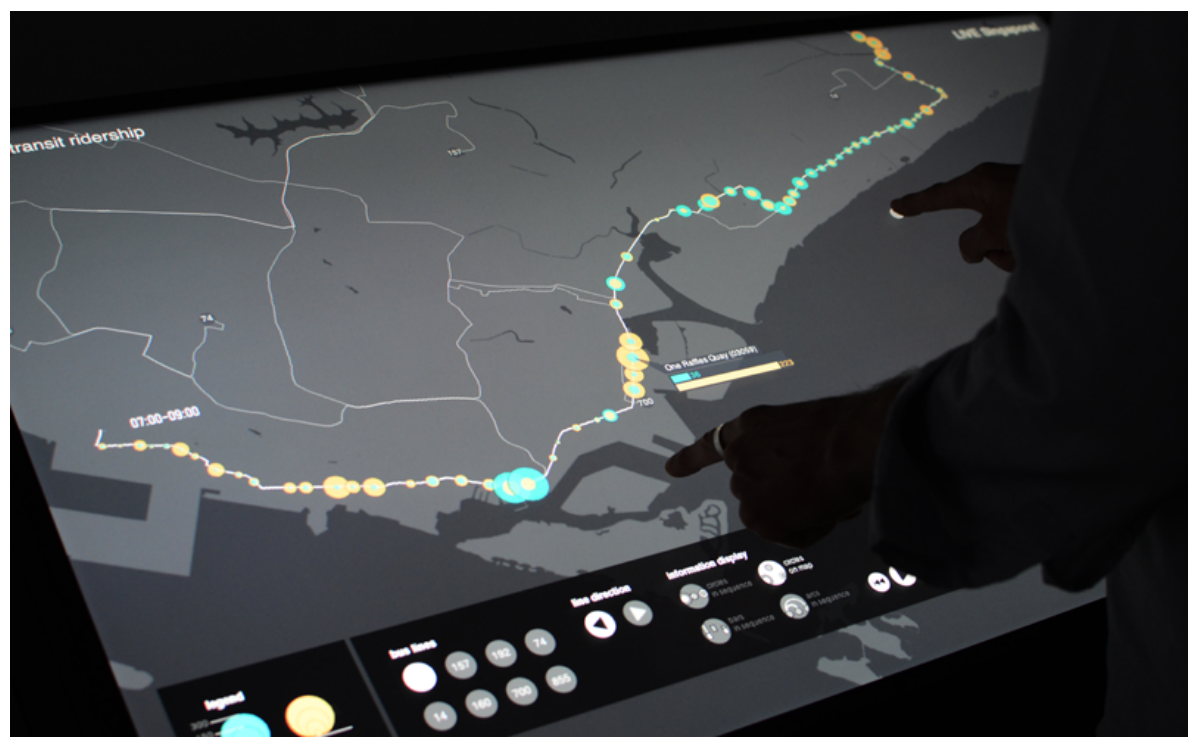

Fig. 3. Visualization of public transit ridership in Singapore, using Unfolding's built-in multitouch interactions for map manipulations

Live Singapore. A visualization of public transit ridership in Singapore [29]. It shows bus passenger flows in three coordinated visualizations (map, time chart, arc diagram), and allows users to interactively explore bus lines and areas of interest (see Fig. 3). Unfolding was used for the map view and for the display of the geo-spatial data glyphs. Interactions with the map are restricted to the city state of Singapore, i.e. when an end user pans or zooms outside of that area, the map gently animates back. One of the challenges in developing this visualization was to create a performant data display method in order to keep high responsiveness on every user interaction. End users can slide through the time dynamically which is directly reflected in the geo-spatial markers. Unfolding supports traversing the visualization pipeline in an efficient way, so that after users adapt the time range the data gets newly aggregated and displayed nearly instantaneously. 


\subsection{Design rationale}

In this section we explain the reasoning for our design decisions in developing Unfolding.

Simplified Java dialect. Processing is a programming language to create interactive graphics, which is used for learning, prototyping, and production [22], and "targets an audience of computer-savvy individuals who are interested in creating interactive and visual work through writing software but have little or no prior experience" [30]. It has a large and active community, with many libraries providing particular additional functionality if needed. Furthermore, Processing is beneficial for more advanced developers: in comparison to visualization libraries which often use high level programming languages, and an elaborate component structure, Processing provides a low level graphic based environment. The flexibility to investigate and develop new visualization and interaction techniques usually requires relatively low level programming and considerable development time [9]. With Unfolding we aim to support this flexibility while reducing the complexity.

One drawback for more advanced developers is the very simple editor (due to the aim of not overwhelming beginners), with nearly no features of modern Integrated Development Environments (IDE), such as code assistance. To circumvent this, Unfolding provides its library for Processing, as well as for full Java IDEs such as Eclipse.

Tile-based. Users know and expect the interaction possibilities of online maps. Tile-based maps are a established way of providing zoom and pan functionality. It furthermore enables to select from a huge range of existing map styles, or customize styles with existing tools. The library uses the so-called Slippy Map technique [23], which uses a tile-based algorithm with pre-rendered map tiles for fixed geographical locations in different provided zoom levels. This is used widely for online web map services (e.g. Google Maps), and custom map styling applications (such as CloudMade [24], TileMill [31]). While map tiles technically support other tile sizes or other map projections, typically the same size of $256 \mathrm{x} 256 \mathrm{px}$, and the same Spherical Mercator projection is used. This restricts geovisualizations to a subset, but simplifies the handling. By using such tiles, non-GIS-experts can easily use existing web tiles or custom map styles, and not care about an own map server stack. 
Desktop-based. For creating sophisticated geovisualization applications, i.e. for big sets of data, or creating multitouch interactions for exhibitions, the use of the Java based programming language Processing includes the ability to use OpenGL for high performance visualizations of tens of thousands of visual elements. While web technology such as WebGL more and more includes these abilities, it still needs more advanced programming skills, and extensive knowledge of the newest browser developments at the moment. Another reason for using a desktop based programming language is the ability to employ large-scale interfaces, such as visualizations on interactive multitouch tabletops.

Simple software architecture. While one of the principles of Processing and many Processing libraries is to provide most methods in a single class for easier access, this comes with a cost: the API itself becomes unstructured and bloated, and the functionality more complex to extend. Similarly, visualization libraries offer lots of functionality, and while they can be extended it tends to be difficult. This is due to the complex software architecture, where new components need to adhere to the sophisticated class structure. The advantage is that - after learning the deeper parts of the API and implementing new features correctly an integrated component can profit from existing mechanisms, e.g. interaction or transition patterns. In Unfolding, we intended combining the simplicity of Processing with proven design patterns in software architecture to achieve the extensibility of other libraries. One of our aims was to create a clear Unfolding API enabling beginners to create own sketches showing geo-spatial data, while at the same time allowing more advanced developers to enhance functionality in a reusable way.

Documentation. To support good learnability, the library comes with extensive documentation, mostly in the form of tutorials and example code. The basic API documentation comes in standard JavaDoc format, and describes the methods of Unfolding. We followed Robillard [32], who proposed to use examples, and categorized them in snippets (short code examples), tutorials (code examples with prose), and applications (longer code examples from actual applications). We distribute various examples in the Unfolding library. On the website we additionally publish tutorials and example applications, so beginners can use or copy these code samples directly in their sketches. 


\subsection{Summary}

In summary, Unfolding provides functionality to handle geo-spatial data and display them on interactive tile-based maps by using reusable components in Processing. Unfolding is not just a collection of existing visualizations; it provides the foundation to create interactive maps, and a basic set of reusable components for building customized or novel geovisualizations.

\section{Evaluation}

In this section we demonstrate the usefulness of Unfolding by presenting selected projects, and describing our user survey and its results. We also give some numbers indicating the library's acceptance.

\subsection{Applications}

We follow the argument of the authors of the widespread Protovis visualization library that one of the main values of a toolkit is in the design and dissemination of successful visualizations [33]. We collected 40 projects which were publicly accessible on the web and referred to the Unfolding website, or were described in publications. From these, we selected notably successful projects as examples for each of the three task groups.

Learning. Student projects have won student competitions (Tweetography [34] is the Winner of the Harvard Conant Prize for "Best NonTraditional Project", and Foreign Domestics [35] is one of the winners of the Visualizing Marathon 2012.), or have been featured in design magazines (LiquiData [36] in Weave magazine [37]).

Prototyping. An example of using Unfolding as a prototyping tool to quickly analyze data-sets is an animation of viewers of TED talks [38]. Various research projects have employed Unfolding to create interactive prototypes to be able to develop and evaluate novel visualization and interaction methods (e.g. [39], [29]). In a visualization for exploring geospatial networks a new interaction technique for solving the fat-finger problem was introduced. Their user study showed that end users could casually interact with the system and were satisfied with the ease of use of this multitouch visualization [29].

Creating. In the last group, successful design projects were publicly exhibited (e.g. Max-Planck-Research Networks [28], a visualization of research networks on a multitouch table, or The Quiet Walk, a system for sonic exploration of urban space [40]). A commercial project for visually 
analyzing tax-free sales on an airport [41] has been featured in Cairo's book on visualization [42].

We believe these Unfolding applications demonstrate compelling realworld usage.

\subsection{Dissemination}

Unfolding was publicly released in August 2011, and the first public version (0.8) was downloaded over 3000 times in the following twelve months. The next version (0.9) was published end of September 2012 and downloaded over 2200 times in the first three months (as per 31st December 2012). While these numbers are just a single measurement, it indicates that Unfolding is widely used, and well accepted. (For instance, the authors of the Prefuse library mention in [16] it had been downloaded 1300 times after the alpha-release.)

\subsection{User survey}

We ran a user study as an online survey after the design and implementation of the second release of Unfolding. The purpose of the survey was to gather feedback on library and feature usage, and measure satisfaction on several aspects such as learnability and suitability. A secondary intention was to gather feedback in order to further improve Unfolding.

Survey design. The questionnaire consisted of sections on the participant's background and prior experience, on the projects they used Unfolding for, and on their satisfaction with the library's features and use.

The survey is partly based on an ISO standard to evaluate software quality [43], and partly on the System Usability Scale (SUS) to collect the subjective rating of the library's usability [44]. We adapted the phrasing in order to have precise yet not overly formal questions. The drawback is that we did not adhere to the standard, and would not be able to compare our results with the usability of other systems. As we have not found other studies on visualization libraries using SUS, we deemed this as acceptable. We mainly tried to keep the survey form brief. We encouraged participants to give comments and constructive criticism, by providing free-form text fields with open questions (e.g. "Do you have any suggestions on how to improve Unfolding?"). All these aspects were based on recommendations to increase response rate in online surveys [45]. 
We used a 5-point Likert scale for satisfaction (ranging from "Highly satisfied" to "Not at all satisfied"), and for agreement to given statements (ranging from "Strongly agree" to "Strongly disagree"). Overall, the survey contained 12 multiple choice, 7 Likert-scale grid, and 6 open questions. Test participants from our group needed circa 15 minutes to fill out the complete form.

The survey was designed as an online questionnaire ${ }^{6}$, was accessible under a public URL, and ran for 10 days in early December 2012. All responses were anonymous.

Participants. As our intention was to gather feedback from persons familiar with Unfolding, we chose library users as potential participants. These persons identified themselves by being active in the Unfolding community, be it on the forum, having published their Unfolding projects online, or having contacted us with questions before. We invited 93 persons via e-mail, from which 32 participated ( $34 \%$ response rate). This of course means we did not collect feedback from developers who decided against Unfolding, which might have biased the satisfaction results. However, we announced the survey on the Unfolding website and in the Processing community forum, via which we received another 5 responses. Overall, this resulted in a total of 37 survey submissions.

Participants were from all age groups (16\% under 24, 44\% 25-34, 31\% $35-44,9 \%$ over 45 years), and nearly half of them students (41\%). They stated their expertise mainly in Design (21 participants) and Visualization (18 p.), with Software Development (15 p.), Data (10 p.) and GIS (2 p.) as runner-ups (participants could enter more than one area). They selfassessed their skill level mostly not as novices, with 25\% expert, $41 \%$ advanced, $25 \%$ intermediate, and $9 \%$ beginner skills.

Survey Results. In the following, we present how satisfied participants were with Unfolding's usability and features, and discuss some further results.

Participants were mostly highly satisfied or satisfied with Unfolding's learnability, understandability, and suitability (see box plots in Fig. 4). They also agreed to the statements that Unfolding is feature rich, and has a well designed API (Fig. 5).

Nearly all participants were highly satisfied or satisfied with Unfolding's basic features (such as displaying maps (97\%), or enabling zoom and

\footnotetext{
${ }^{6}$ Find the full survey archived at http://unfoldingmaps.org/survey-2012-archived.pdf, and the original results at http://unfoldingmaps.org/survey-2012-results.pdf
} 


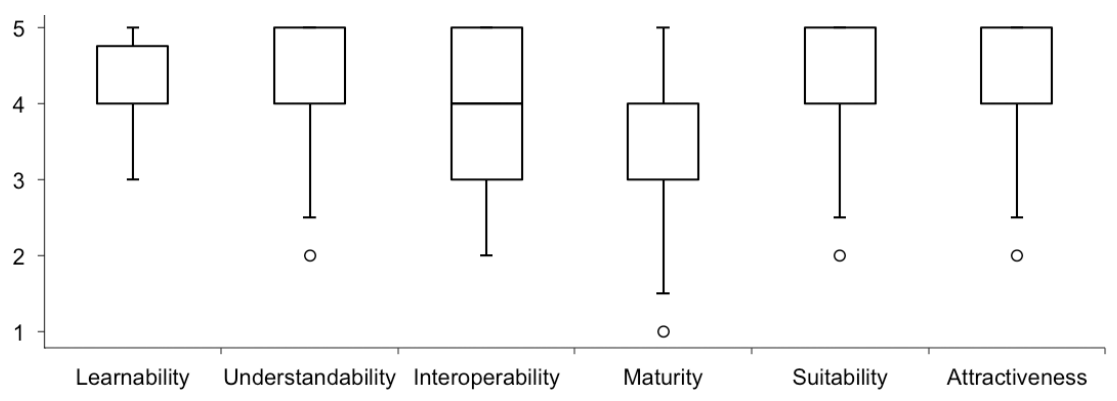

Fig. 4. Satisfaction with Unfolding

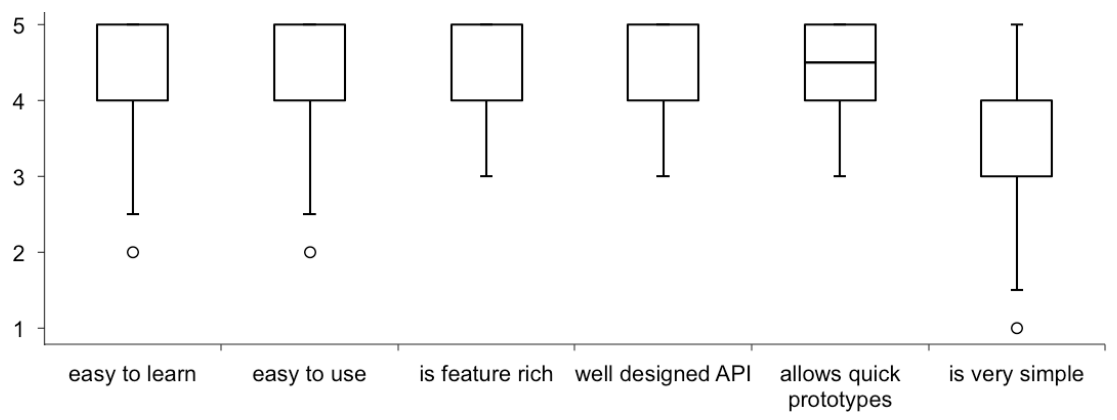

Fig. 5. Agreement with statements

pan interaction (91\%)). However, fewer participants were satisfied with more advanced functionality such as displaying labels $(51 \%)$ or loading geo-spatial data (54\%). While these numbers still indicate a majority of users being satisfied, we assume a connection to the documentation of these more advanced features.

Most participants were highly satisfied or satisfied with examples $(62 \%)$ and tutorials (53\%). This reflects our decision of focusing on these sections for learning Unfolding (cf 4.3). However, participants were not fully satisfied with the API documentation (38\% highly satisfied or satisfied). This suggests that even though studies have shown developers use examples and tutorials to learn a new API, and re-use existing code snippets to quickly create own prototypes [32], library users expect a complete and well-written interface description, in any case. Three participants suggested improving the documentation in our general free-form comment field.

More than half the participants had some prior experience with GIS software (19\% use it often, $19 \%$ occasionally, and $22 \%$ at least once). Besides the Google Maps API (57\%) few have used other map libraries 
often or occasionally (9\% Leaflet, $12 \%$ Open Layers, $12 \%$ PolyMaps, $15 \%$ ModestMaps). However, around one third or more have used these libraries at least once (28\% L, 40\% OL, 31\% PM, $46 \%$ MM, respectively). To the question why Unfolding was chosen over other libraries, free-form responses included the Processing environment (e.g. "well integrated with Processing"), and the ability for quick prototyping (e.g. "it's quite easy to get results quickly", "It allowed me to dump geo data directly on a map in less than an hour"). Three participants named the integration with TileMill as reason. We did not expect the latter, as other libraries also allow this. We assume this is due to a tutorial about Unfolding's TileMill functionality which was linked on well-known visualization blogs.

Participants used Unfolding for visualizations ranging from student to research to commercial projects. They achieved what they planned $(81 \%$ agreed or strongly agreed), and found Unfolding to be helpful in doing so $(87 \%)$.

Overall, participants were highly satisfied $(53 \%)$ or satisfied $(38 \%)$ with the library. Most participants (88\%) plan to use Unfolding in the future (with $6 \%$ not, and the rest don't know).

\section{Conclusion}

We presented the Unfolding library to create interactive maps and geovisualizations. Both creating our own applications, as well as collecting feedback from visualization projects by others has helped us to adapt the library, and to repeatedly refine its function range. The results from our user survey prove that Unfolding achieved our design goals. Most participants were highly satisfied or satisfied with our library. We see the use in various courses, in student, research and commercial projects as further indicator for the learnability and usability of the library.

Overall, we have shown that Unfolding is beneficial for learning, prototyping, and creating interactive maps and geovisualizations.

\section{Acknowledgments}

We like to thank Felix Lange, and all other library contributors. We also thank the users of Unfolding, especially students from FH Potsdam and from IUAV University of Venice for their feedback, and the participants of the survey. And we like to thank the anonymous reviewers for their helpful feedback. 


\section{References}

1. Heidmann, F.: Interaktive Karten und Geovisualisierungen. In Weber, W., Burmester, M., Tille, R., eds.: Interaktive Infografiken. Springer, Heidelberg (2013) $43-74$

2. Dodge, M., Perkins, C., Kitchin, R.: Mapping modes, methods and moments. In Dodge, M., Perkins, C., Kitchin, R., eds.: Rethinking Maps: New frontiers in cartographic theory. Routledge, London (2009) 220-243

3. Antonelli, P.: States of Design 01: Visualization. Domus 946 (April 2011)

4. Dykes, J., Andrienko, G., Andrienko, N., Paelke, V., Schiewe, J.: GeoVisualization and the Digital City. Computers, Environment and Urban Systems 34(6) (2010) 443-451

5. Andrienko, G., Andrienko, N., Demsar, U., Dransch, D., Dykes, J., Fabrikant, S., Jern, M., Kraak, M., Schumann, H., Tominski, C.: Space, time and visual analytics. International Journal of Geographical Information Science 24(10) (2010) 15771600

6. Wisniewski, P., Pala, O., Lipford, H., Wilson, D.: Grounding geovisualization interface design: a study of interactive map use. In: Proceedings of the SIGCHI conference on Human Factors in Computing Systems Extended Abstracts, ACM (2009) 3757-3762

7. Jones, C., Haklay, M., Griffiths, S., Vaughan, L.: A less-is-more approach to geovisualization-enhancing knowledge construction across multidisciplinary teams. International Journal of Geographical Information Science 23(8) (2009) 1077-1093

8. Batty, M., Hudson-Smith, A., Milton, R., Crooks, A.: Map mashups, Web 2.0 and the GIS revolution. Annals of GIS 16(1) (2010) 1-13

9. Wood, J., Dykes, J., Slingsby, A., Clarke, K.: Interactive visual exploration of a large spatio-temporal dataset: reflections on a geovisualization mashup. Visualization and Computer Graphics, IEEE Transactions on 13(6) (2007) 1176-1183

10. IndieMapper. http://indiemapper.com/

11. GeoCommons. http://geocommons.com/

12. Hardisty, F., Robinson, A.: The geoviz toolkit: using component-oriented coordination methods for geographic visualization and analysis. International Journal of Geographical Information Science 25(2) (2011) 191-210

13. Turton, I.: Geo Tools. In Hall, G., Leahy, M., eds.: Open Source Approaches in Spatial Data Handling. Volume 2 of Advances in Geographic Information Science. Springer, Heidelberg (2008) 153-169

14. DataVisualization.ch Selected Tools. http://selection.datavisualization.ch

15. Bostock, M., Ogievetsky, V., Heer, J.: $D^{3}$ Data-Driven Documents. Visualization and Computer Graphics, IEEE Transactions on 17(12) (2011) 2301-2309

16. Heer, J., Card, S., Landay, J.: Prefuse: a toolkit for interactive information visualization. In: Proceedings of the SIGCHI conference on Human factors in computing systems, ACM (2005) 421-430

17. Leaflet. http://leaflet.cloudmade.com/

18. PolyMaps. http://polymaps.org/

19. GeoMap. http://www.gicentre.org/geomap/

20. Google Mapper. http://googlemapper.pt.vu/

21. Modest Maps. http://modestmaps.com/

22. Reas, C., Fry, B.: Processing: programming for the media arts. AI \& Society 20(4) (2006) 526-538 
23. Haklay, M., Weber, P.: Openstreetmap: User-generated street maps. Pervasive Computing, IEEE 7(4) (2008) 12-18

24. CloudMade. http://cloudmade.com/

25. Harrower, M., Sheesley, B.: Designing Better Map Interfaces: A Framework for Panning and Zooming. Transactions in GIS 9(2) (2005) 77-89

26. You, M., Chen, C., Liu, H., Lin, H.: A usability evaluation of web map zoom and pan functions. International Journal of Design 1(1) (2007) 15-25

27. Tidwell, J.: Designing interfaces. O'Reilly Media (2010)

28. Max Planck Research Networks. http://max-planck-research-networks.net/

29. Nagel, T., Duval, E., Vande Moere, A.: Interactive exploration of geospatial network visualization. In: Proceedings of the SIGCHI conference on Human Factors in Computing Systems Extended Abstracts, ACM (2012) 557-572

30. Reas, C., Fry, B.: Processing: a programming handbook for visual designers and artists. Mit Press (2007)

31. TileMill. http://tilemill.com/

32. Robillard, M.: What makes APIs hard to learn? Answers from developers. Software, IEEE 26(6) (2009) 27-34

33. Bostock, M., Heer, J.: Protovis: A graphical toolkit for visualization. Visualization and Computer Graphics, IEEE Transactions on 15(6) (2009) 1121-1128

34. Patel, S., Sun, W. http://tweetography.herokuapp.com

35. Foreign Domestics. http://visualizing.org/visualizations/ foreign-domestics

36. LiquiData. http://liquidata.org/en/

37. Kiefer, C., Nagel, T.: Neue Sichtbarkeit. Weave magazine (6) (2011)

38. TED Talks - A Visual Map. http://blog.ted.com/2012/09/28/ who-else-is-watching-tedtalks-a-visual-map/

39. Nagel, T., Heidmann, F., Condotta, M., Duval, E.: Venice Unfolding: a tangible user interface for exploring faceted data in a geographical context. In: Proceedings of the 6th Nordic Conference on Human-Computer Interaction: Extending Boundaries, ACM (2010) 743-746

40. Altavilla, A., Tanaka, A.: The Quiet Walk: Sonic Memories and Mobile Cartography. In: Proceedings of the 9th Sound and Music Computing Conference. (2012)

41. Tax Free Retail Analysis Tool. http://tulpinteractive.com/projects/ tax-free-retail-analysis-tool/

42. Cairo, A.: The Functional Art: An introduction to information graphics and visualization. New Riders (2012)

43. ISO 25010: Systems and Software Quality Requirements and Evaluation (2011)

44. Brooke, J.: SUS-A quick and dirty usability scale. Usability evaluation in industry 189 (1996) 194

45. Nulty, D.: The adequacy of response rates to online and paper surveys: what can be done? Assessment \& Evaluation in Higher Education 33(3) (2008) 301-314 